\title{
Current Development of Flow Visualization: Techniques and Methods
}

\author{
Abdullah Bade and Danny Ngo Lung Yao* \\ Faculty of Science and Natural Resources (FSSA), University Malaysia Sabah (UMS), \\ Jalan UMS, 88400 Kota Kinabalu, Sabah, Malaysia
}

\begin{abstract}
Flow visualization is commonly used in studying fluid dynamics since fluids such as air, water and wind have invisible flow patterns due to its transparent property. In this era of big data, flow visualization is important to provide a compelling method of exploring, analysing and presenting large-scale flow field data. Flow visualization provides better insight into the fluid properties in space and time. This paper intends to provide an overview on the development of flow visualization methods such as spot noise, line integral convolution (LIC), texture advection, streamline placement, etc. from early 2000 to recent years. The reviewed methods will be compared and evaluated to determine the optimal approach of flow visualization.
\end{abstract}

Keywords: Flow visualization, line integration convolution, texture advection

\section{INTRODUCTION}

Recently, many studies focus on flow visualization to understand the fluid dynamics. Fluids are basically transparent which causes the flow pattern becomes invisible. Thus, many studies had been carried out to visualize the flow patterns. There is four major group in the study of flow visualization such as direct flow visualization, particle tracing methods, texture-based flow visualization, and feature-based flow visualization. This paper attempted to review the recent development of the flow visualization methods from early 2000 to recent years.

\section{DIRECT FLOW VISUALIZATION}

Direct flow visualization usually used glyphs and mapping to directly map data graphically.

Figure 1 shows the flow visualization using glyphs. Volume rendering method was commonly used in visualizing the $3 \mathrm{D}$ flow. The thermal flow visualization in an automotive cabin (Ono et al., 2001) is an application study of visualizing $3 \mathrm{D}$ flow data by using the volume rendering method. Unlike the classic glyphs that display direction, flow radar glyphs (Hlawatch et al., 2011) integrated the idea of radar display to visualize unsteady flow where the direction and time are displayed by angle and spherical coordinates respectively.

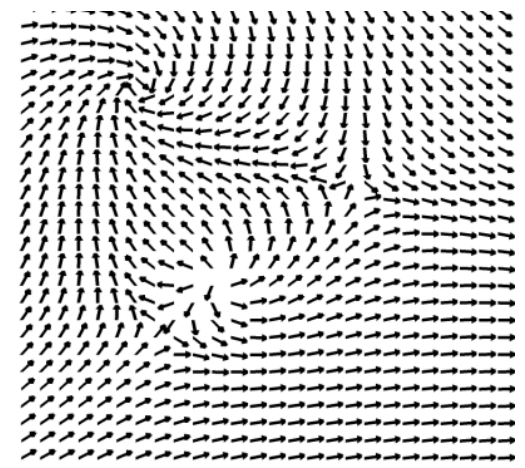

Figure 1. Glyphs.

\section{PARTICLE TRACING METHODS}

Particle tracing methods used particle tracer to obtain the flow pattern. Streamline, streakline, pathline, and timeline are commonly used in particle tracing to display

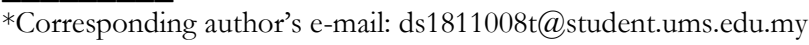


the flow patterns. Streamline had been extended as evenly spaced streamline (Jobard \& Lefer, 2000) to visualize $2 \mathrm{D}$ steady flow based on the computational of temporal series of correlated images. Multiresolution flow visualization (Jobard \& Lefer, 2001) integrated evenly spaced streamline with a multiresolution theory to produce different densities of streamline-based images. Multiresolution unsteady flow visualization (Ueng \& Sun, 2007) proposed to interpolate the flow fields in the time domain based on special Navier Stoke equations. Evenly spaced streamline also being combined with glyphs in wind visualization (Pilar \& Ware, 2013) where streamline can better display the flow patterns and glyphs represent wind direction. Figure 2 shows the combination of evenly spaced streamline and glyphs.

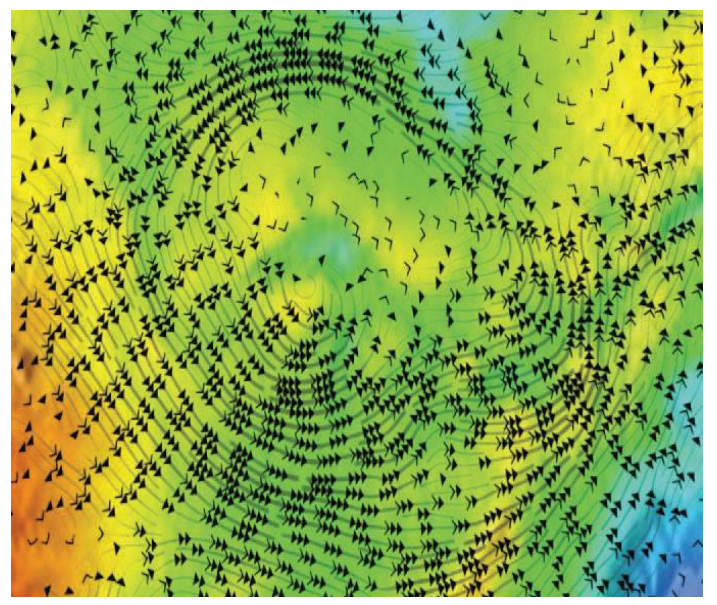

Figure 2. Arrow glyphs on evenly spaced streamline (Pilar \& Ware, 2013)

Similarity guided streamline placement (Chen et al., 2007) attempted to avoid redundant streamlines by measuring streamline proximity based on a similarity metric. Illustrative streamline placement (Li et al., 2008) used a dissimilarity metric to decide whether the new seed needs to be placed or not at the local point. Flow topology emphasized streamline placement (Zhang et al., 2010) was able to highlight topological structure by seeding the streamline in a subfield where the subfield will be subdivided and placed new seed until there no empty subfield. Multiresolution streamline placement (Zhang et al., 2011) proposed to visualize 2D flow at the different level of details by distributing streamlines based on the constructed control grids' hierarchy. Parallel streamline placement (Zhang et al., 2013) used the concept of Local Tracing Areas (LTAs) to place the streamline in parallel.

Flow partition method (Chen \& Fujishiro, 2008) attempted to optimize the parallel performance of streamline on large scale data. Streamline illumination method (Zhang et al., 2013) improved the spatial perception of streamline by computing the line illumination based on the Phong/Blinn model. Centroidal Voronoi Tessellation (CVT) (Liu et al., 2013) had been applied to streamline so that streamlines can be like geometric characteristics of the flow field. Figure 3 shows the difference between ordinary Voronoi tessellation and CVT. Focus + Context streamline (Tao et al., 2014) was able to reduce occlusion and clutter around focal regions. Probabilistic streamline (He et al., 2016) used a Bayesian approach to generate streamlines in uncertain flow more accurately. High order access dependencies-based method (Zhang et al., 2016) enhanced pathline computation by tracing pathlines in both forward and backward directions.

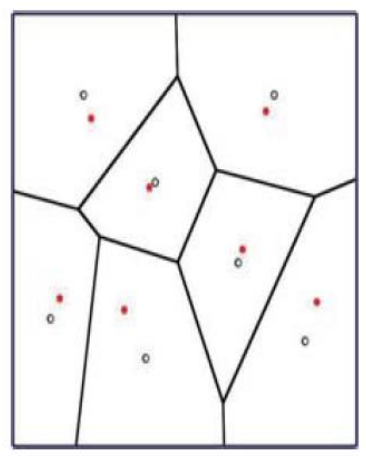

(a)

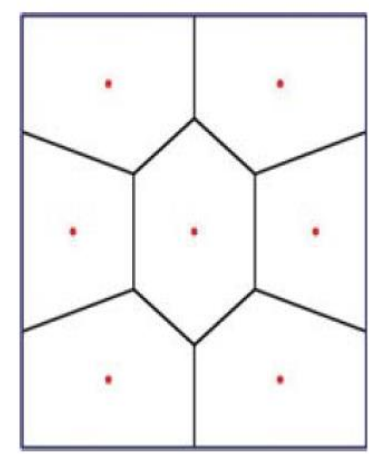

(b)
Figure 3. (a) Ordinary Voronoi Tessellation. (b) CVT (Liu et al., 2013)

\section{TEXTURE BASED FLOW VISUALIZATION}

ITexture-based flow visualization usually presents the flow densely using generated noise texture. Figure 4 shows the example of texture-based flow visualization. The common approaches to texture-based flow visualization include spot noise, LIC and texture 
advection. Spot noise generally generates noise texture with spots that represent moving particles. GPU based spot noise (Qin et al., 2010) computes the spot noise in parallel to accelerate the 2D flow visualization.

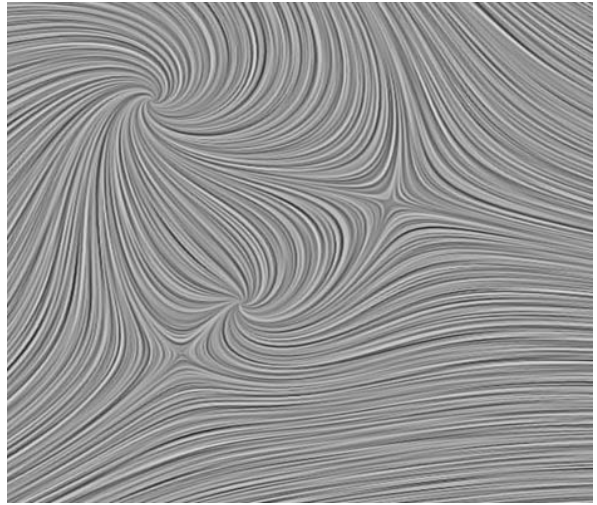

Figure 4. Texture-based flow visualization.

LIC was an effective approach for visualizing $2 \mathrm{D}$ dense flow field by generates noise texture by filtering using convolution with a filter kernel. Hardware accelerated selective LIC volume rendering (Suzuki et al., 2002) was attempted to extend LIC to $3 \mathrm{D}$ by using scalar volume rendering hardware, VolumePro based on 3D S-map construction and volume illumination model. Feature emphasized oriented LIC (Liu et al., 2013) generated noise texture with non-uniform streamlets to highlight the flow features. Figure 5 showed the difference between LIC and feature emphasized OLIC whereas LIC can display direction and orientation of flow and feature emphasized OLIC highlighted the vortexes and saddle points.

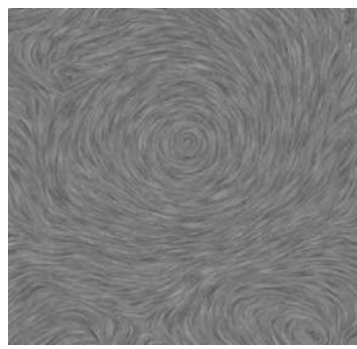

(a)

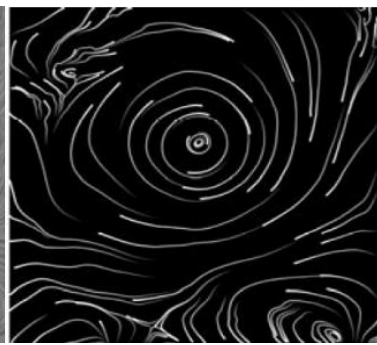

(b)
Figure 5. (a) LIC. (b) Feature Emphasized OLIC (Liu et al., 2013)

Dynamic LIC (Sundquist, 2003) visualized the motion of streamline-based on the motion vector field. Output-coherent image space LIC (Huang et al., 2012) can preserve the texture coherence while visualizing the surface flows. Output-sensitive 3D LIC (Falk \& Weiskopf, 2008) was able to visualize large data as the rendering speed independent of data size. Flow direction reflection problem had been solved by integrating LIC with a cool or warm tone transformation (Kai et al., 2010). Projected 2D LIC (Toledo \& Celes, 2011) extend the LIC to the $3 \mathrm{D}$ arbitrary surface. Seed LIC volume rendering (Helgeland \& Andreassen, 2004) can visualize $3 \mathrm{D}$ flow volume and reveal depth relations using limb darkening.

Unsteady flow line integral convolution (UFLIC) was an approach that visualizes flow with high temporalspatial coherence. Accelerated UFLIC (Liu \& Moorhead II, 2005) used the pathlines reuse strategy to accelerate UFLIC. Parallel UFLIC (Ding et al., 2015) implemented UFLIC in parallel to provide a high performance on the large time varying flow visualization. UFLIC also applicable on the closest point embedding surface (Kim \& Hansen, 2015). Orthogonally Gabor-Enhanced Repetitive LIC (OGR LIC) (Matvienko \& Krüger, 2015) can produce a frequency-oriented image by allowing explicit frequency control.

Texture advection had been extended to hardwareaccelerated texture advection (Jobard et al., 2000) and 3D flow (Kao et al., 2001). Anisotropic diffusion method (Diewald et al., 2000) had been implemented to visualize Euclidean domains and surfaces. Later, anisotropic diffusion method had been extended for a multiscale visualization of long-time, complex transport problem (Burkle et al., 2001). Lagrangian-Eulerian Advection (LEA) (Jobard et al., 2002) used the Lagrangian scheme and Eulerian scheme to integrates particle position and advects particle colour respectively. Figure 6 shows the flowchart of LEA. Later, a hardware-accelerated LEA (Weiskopf et al., 2002) allowed every texture advection can be completed in a singlepass rendering. LEA was later extended into Lagrangian-Eulerian time surfaces (Grant et al., 2002) to visualize the ocean flow vertical motions. 


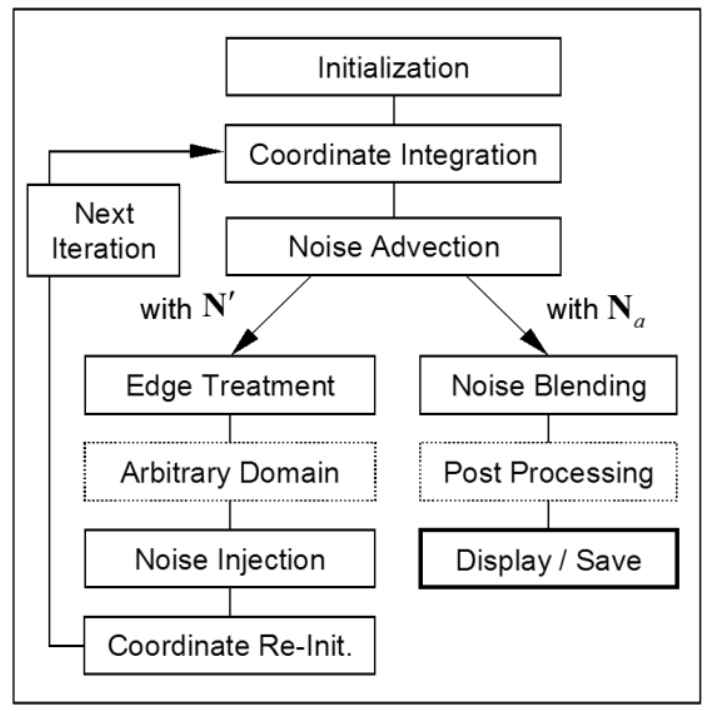

Figure 6. Flowchart of LEA.

Image-based flow visualization (IBFV) (van Wijk, 2002) can produce a variety of visualizations based on advection and decay of dye. 3D IBFV (Telea \& van Wijk, 2003) extends the IBFV into visualizing $3 \mathrm{D}$ flow by using the hardware-accelerated method. IBFV also extended to visualize for curved surfaces (van Wijk, 2003). Image Space Advection was a combination of LEA and IBFV that can visualize unsteady flow with high spatiotemporal correlation (Laramee et al., 2003).

\section{FEATURE BASED FLOW VISUALIZATION}

Feature-based flow visualization usually exhibits the flow sparsely based on the flow features such as vortices. The topology-based method usually emphasized only flow features but incapable of many critical points. Thus, the topology-based method had been improved by simplification (Tricoche et al., 2000), continuous simplification (Tricoche \& Scheuermann, 2001), compression (Lodha et al., 2000) and smoothing (Westermann et al., 2001). Galilean invariant extraction (Sahner et al., 2005) was a vortex core extraction method that extracts ridge and valley lines of Galilean invariant vortex region. Rotation invariant extraction (Güntheret al., 2016) can extract the vortex that under uniform speed rotation by transforming Galilean invariance to rotation invariance. Figure 7 show the result of Galilean invariant and rotation invariant on vortex cores of centrifugal pump.
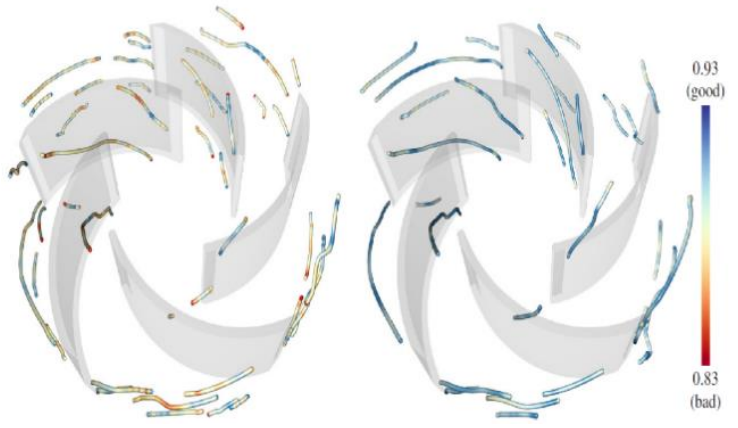

Figure 7. (a) Galilean Invariant and (b) rotation invariant.

Maxima score (Ferrrari \& Hu, 2014) can extract the flow features by calculating the normalized maxima score based on a given point's scalar intensity relative to its neighbours. Feature-based visualization algorithm (Liang et al., 2010) was able to visualize large-scale flow data by using spiral strategy.

\section{DISCUSSION}

Direct flow visualization not suitable for complex data as glyphs can display direction better but will have discontinues between glyphs. Particle tracing methods can display flow patterns without any discontinuity but have no direction. However, the problem solved as streamline had proved its ability to combine with glyphs. Texture-based flow visualization is highly flexible where LIC, spot noise, glyphs can be combined to better display the flow data.

The current challenge on flow visualization are the complexity of $3 \mathrm{D}$ flow field data, time-varying properties and the compatibility of current method on large scale 3D unsteady flow visualization.

\section{ACKNOWLEDGEMENT}

The authors would like to thank the support of GPRL UM 


\section{REFERENCES}

Burkle, D., Preusser, T. \& Rumpf, M. (2001). Transport and anisotropic diffusion in timedependent flow visualization. Proceeding Visualization, 61-67.

Chen, L. \& Fujishiro, I. (2008). Optimizing parallel performance of streamline visualization for large distributed flow datasets. IEEE Pacific VIS, 8794.

Chen, Y., Cohen, J. \& Krolik, J. (2007). Similarityguided streamline placement with error evaluation. IEEE Transactions on Visualization and Computer Graphics, 13, 6, 1448-1455.

Diewald, U., Preusser, T., \& Rumpf, M. (2000). Anisotropic diffusion in vector field visualization on Euclidean domains and surfaces. IEEE Transactions on Visualization and Computer Graphics, 6, 2, 130-149.

Ding, Z., Liu, Z., Yu, Y. \& Chen, W. (2015). Parallel unsteady flow line integral convolution for highperformance dense visualization. IEEE Pacific Vis, 25-30.

Falk, M. \& Weiskopf, D. (2008). Output-sensitive 3D line integral convolution. IEEE Transaction on Visualization and Computer Graphics, 14, 4, 820834 .

Ferrrari, S. \& Hu, Y. (2014). Using maxima score for the extraction and visualization of fluid flow structures. $11^{\text {th }}$ International Conference on Computer Graphics, Imaging and Visualization, 96-100.

Grant, J., Erlebacher, G. \& O’Brien, J. (2002). Case study: visualizing ocean flow vertical motions using Lagrangian-Eulerian time surfaces. IEEE Visualization, 529-532.
Günther, T., Schulze, M. \& Theisel, H. (2016). Rotation invariant vortices for flow visualization. IEEE Transactions on Visualization and Computer Graphics, 22, 1, 817-826.

He, W., Chen, C.-M., Liu, X. \& Shen, H.-W. (2016). A Bayesian approach for probabilistic streamline computation in uncertain flows. IEEE Pacific Vis, 214-218.

Helgeland, A. \& Andreassen, O. (2004). Visualization of vector fields using seed LIC and volume rendering. IEEE Transactions on Visualization and Computer Graphics, 10, 6, $673-682$

Hlawatsch, M., Leube, P., Nowak, W. \& Weiskopf, D. (2011). Flow-radar glyphs - static visualization of unsteady flow with uncertainty. IEEE Transactions on Visualization and Computer Graphics, 17, 12, 1949-1958.

Huang, J. Pei, W., Wen, C., Chen, G., Chen, W. \& Bao, H. (2012). Output-coherent image-space LIC for surface flow visualization. IEEE Pacific Vis, 137-144.

Jobard, B., Erlebacher, G. \& Hussaini, M.Y. (2000). Hardware-accelerated texture advection for unsteady flow visualization. IEEE Visualization, 155-162.

Jobard, B., Erlebacher, G. \& Hussaini, M.Y. (2002). Lagrangian-Eulerian advection of noise and dye textures for unsteady flow visualization. IEEE Transactions on Visualization and Computer Graphics, 8, 3, 211-222. 
Jobard, B. \& Lefer, W. (2000). Unsteady flow visualization by animating evenly-spaced streamlines. Eurographics, 31-40.

Jobard, B. \& Lefer, W. (2001). Multiresolution flow visualization. WSCG Conference Proceedings, 3437.

Kai, Y., Hua-xun, X., Xun, C. \& Si-kun, L. (2010). A LIC method based on cool/warm tone. ICALIP, 239-243.

Kao, D., Zhang, B., Kim, K. \& Pang, A. (2001). 3D Flow visualization using texture advection, IASTED Conference on Computer Graphics and Imaging 01 (CGMI), 252-257.

Kim, M. \& Hansen, C.D. (2015). Surface flow visualization using the closest point embedding. IEEE PacificVis,17-23.

Laramee, R.S., Jobard, B. \& Hauser, H. (2003). Image space based visualization of unsteady flow on surfaces. IEEE Visualization, 131-138.

Li, L., Hsieh, H. \& Shen, H. (2008). Illustrative streamline placement and visualization. IEEE Pacific Vis, 79-86.

Liang, Z., He, C.T. \& Xin, Z. (2010). Feature based visualization algorithm for large scale flow data. Second International Conference on Computer Modeling and Simulation, 1, 194-197.

Liu, W., Lu, L., Lévy, B., Yang, C. \& Meng, X. (2013). Centroidal Voronoi Tessellation of streamlines for flow visualization. $10^{\text {th }}$ International Symposium on ISVD, 76-81.

Liu, Z. \& Moorhead II, R.J. (2005). Accelerated unsteady flow line integral convolution. IEEE Transactions on Visualization and Computer Graphics, 11, 2, 113-125.

Liu, B., Zhang, W., Wang, Y. \& Pei, Y. (2013). Feature emphasized OLIC for 2D flow visualization. $9^{\text {th }}$ International Conference on CIS, 635-639.

Lodha, S.K., Renteria, J.C. \& Roskin, K.M. (2000). Topology-preserving compression of $2 \mathrm{D}$ vector fields. IEEE Visualization, 343-350.

Matvienko, V. \& Krüger, J. (2015). Explicit frequency control for high-quality texturebased flow visualization. IEEE Sci Vis, 41-48.

Ono, K., Matsumoto, H. \& Himeno, R. (2001). Visualization of thermal flows in an automotive cabin with volume rendering method. EG/ IEEE

TCVG Symposium on Visualization, 301-308.

Pilar, D.H.F. \& Ware, C. (2013). Representing flow patterns by using streamlines with glyphs. IEEE Transactions on Visualization and Computer Graphics, 19, 8, 1331-1341.

Qin, B., Su, F., Wu, Z. \& Wang, J. (2010). GPU based spot noise parallel algorithm for $2 \mathrm{D}$ vector field visualization. ICOIP, $1,580-583$.

Sahner, J., Wein Kauf, T. \& Hege, H.-C. (2005). Galilean invariant extraction and iconic representation of vortex core lines. EG/ IEEE VGTC Symposium on Visualization.

Sundquist, A. (2003). Dynamic line integral convolution for visualizing streamline evolution. IEEE Transactions on Visualization and Computer Graphics.

Suzuki, Y., Fujishiro, I., Chen, L. \& Nakamura, H. (2002). Case study: hardware-accelerated selective LIC volume rendering. IEEE Visualization, 485-488.

Tao, J., Wang, C., Shene, C. \& Kim, S.H. (2014). A deformation framework for focus + context flow visualization. IEEE Transactions on Visualization and Computer Graphics, 20, 1, 42-55.

Telea, A. \& van Wijk, J.J. (2003). 3D IBFV: hardware-accelerated 3D flow visualization. IEEE Visualization, 233-240.

Tricoche, X. \& Scheuermann, G. (2001). Continuous topology simplification of planar vector fields. IEEE Visualization, 159-166. 
Tricoche, X., Scheuermann, G. \& Hagen, H. (2000). A Zhang, W., Zhang, M. \& Sun, B. (2011). topology simplification method for 2D vector Multiresolution streamline placement for 2D fields. IEEE Visualization, 359-366.

Toledo, T. \& Celes, W. (2011). Visualizing 3D flows of flow fields. Seventh International Conference on CIS, 1174-1178. black-oil reservoir models on arbitrary surfaces using projected $2 \mathrm{D}$ line integral convolution. $24^{\text {th }}$ SIBGRAPI Conference, 133140.

Ueng, S. \& Sun, W. (2007). Multi-resolution unsteady flow visualization. Third International Conference on IIH-MSP, 1, 357-360.

van Wijk, J.J. (2002). Image based flow visualization. ACM Transactions on Graphics, 21, 3, 745-754.

van Wijk, J.J. (2003). Image based flow visualization for curved surfaces. IEEE Visualization, 123-130.

Weiskopf, D., Erlebacher, G., Hopf, M. \& Ertl, T. (2002). Hardware-accelerated LagrangianEulerian texture advection for 2D flow visualization. VMV Conference, 77-84.

Westermann, R., Johnson, C. \& Etrl, T. (2001). Topology-preserving smoothing of vector fields. IEEE Transactions on Visualization and Computer Graphics, 7, 3, 222-229.

Zhang, J., Guo, H. \& Yuan, X. (2016). Efficient unsteady flow visualization with high-order access dependencies. IEEE Pacific Vis, 80-87.

Zhang, W., Pei, Y., Liu, B. \& Guan, M. (2013). A streamline illumination method for $3 \mathrm{D}$ flow fields. Seventh ICIG, 252-257.

Zhang, W., Sun, B. \& Wang, Y. (2010). A streamline placement method highlighting flow field topology. International Conference on CIS, 238242.

Zhang, W., Wang, Y., Zhan, J., Liu, B. \& Ning, J. (2013). Parallel streamline placement for 2D flow fields. IEEE Transactions on Visualization and Computer Graphics, 19, 7, 1185-1198. 